\title{
A case report of inflammatory syndrome presenting as tumor lysis syndrome after single dose of Nivolumab
}

\begin{abstract}
Background: Tumor lysis syndrome (TLS) is an oncologic emergency that is caused by increased turnover of cancer cells. It is often seen with highly proliferative tumors like lymphoma, leukemia, small cell lung cancer or after initiation of therapy. TLS is rarely seen in solid tumors including Renal Cell Carcinomas (RCC) or novel immune therapy agents.

Case presentation: A 74year old Caucasian male with metastatic clear RCC presented to the Emergency Department 2 days after receiving his first dose of Nivolumab. His presentation included progressive weakness, dizziness, and hypotension. Laboratory data confirmed TLS according to the Cairo-Bishop definition. Despite supportive measures, persistent hypotension and tachycardia ultimately lead to his death.
\end{abstract}

Conclusion: This report highlights the potential risk of developing a Tumor lysis Syndrome that is immune related. Such a presentation likely due to a cytokine storm is the first case to be reported.

Keywords: nivolumab, tumor lysis syndrome, renal cell carcinoma, cytokine storm, inflammation
Volume 8 Issue 2 - 2017

\author{
Houssein Abdul Sater, Reena M Patel, Brian T \\ Sullivan, Jigarkumar Parikh \\ Department of Hematology Oncology, Augusta University, USA
}

Correspondence: Houssein A Sater, Hematology Oncology, II 20 I5th Street, BAA-5407, Augusta, Georgia 309|2, USA, Email houssein.md@gmail.com

Received: May 18, 2017| Published: July 17, 2017
Abbreviations: ED, emergency department; ICU, intensive care unit; IVF, intravenous fluids; RCC, renal cell carcinoma; SCCA, squamous cell carcinoma; TLS, tumor lysis syndrome; AST, aspartate transaminase; ALT, alanine transaminase; BUN, blood urea nitrogen; $\mathrm{Cr}$, creatinine

\section{Background}

Tumor lysis syndrome (TLS) is an oncologic emergency that is caused by increased turnover of tumor cells with subsequent release of potassium, phosphate, and uric acid into the systemic circulation. Hyperuricemia, hyperphosphatemia, and calcium phosphate deposition within the renal tubules can lead to acute kidney injury. ${ }^{1}$ TLS is often seen after initiation of therapy in small cell lung cancer or highly aggressive lymphomas and leukemias, such as Burkitts lymphoma and $\mathrm{T}$ cell acute lymphoblastic leukemia. ${ }^{2}$ It is due to the heavy tumor cell burden of these diseases that results in increase cell lysis during chemotherapy. Certain clinical features often predispose to the development of TLS, such as a preexisting kidney disease (including oliguria), state of volume depletion prior or during treatment, and pretreatment hyperuricemia and/or hyperphosphatemia.

Renal cell carcinomas (RCC) are malignant neoplasms involving the renal parenchyma. RCC constitutes more than $90 \%$ of all primary renal neoplasms. ${ }^{4}$ They can present with a variety of symptoms; however, the classic triad of renal cell carcinoma is flank pain, hematuria, and the presence of a palpable abdominal renal mass. Treatment of RCC consists of surgical resection in early stages and palliative targeted therapy in advanced or metastatic setting, mainly using vascular growth factor or VEGF inhibitors. ${ }^{5}$ TLS is rare in this type of tumors with and without treatment.

Nivolumab is a novel immune therapeutic agent manufactured by Ono Pharmaceutical and Medarex approved for the treatment of non- small cell lung cancer and unresectable or metastatic melanoma in patients who no longer respond to conventional therapy. A phase III trial .6 (CheckMate -025) highlighted the efficacy of Nivolumab in metastatic RCC and lead to its FDA approval. Nivolumab acts as an immunomodulator by blocking ligand activation of the programmed cell death 1 (PD-1) receptor on activated T cells. Currently, there are different known adverse effects of Nivolumab reported but TLS and cytokine storm have not been described yet. ${ }^{2}$

The following case report details Nivolumab as a cause of an acute inflammatory or auto-immune process presenting as TLS in a patient with clear cell RCC.

\section{Case presentation}

Here we discuss the case of a 74-year-old Caucasian male with a past medical history significant for chronic kidney disease secondary to type II diabetes mellitus, hypothyroidism, stage II squamous cell carcinoma of the left neck $\mathrm{s} / \mathrm{p}$ modified radical neck dissection and chemo-radiation, and recently diagnosed metastatic clear cell renal cell carcinoma (RCC) s/p right nephroureterectomy in 2013 who presented to the emergency department (ED) with progressive weakness, dizziness, hypotension, and decreased oral intake of solids and liquids 2days after receiving his first dose Nivolumab (240 mg Intravenously). When paramedics arrived to the scene, the patient was noted to be hypotensive and tachycardic with initial exam showing a cachectic male with waxing and waning mentation with otherwise no focal neurologic deficits. Aside from patients vital signs the rest of the exam was noted to be unremarkable. The patient was given approximately 4 liters of fluids in the ED, with improvements in his hypotension and tachycardia. On admission, the patient denied any symptoms of chest pain, dyspnea, nausea, vomiting, diarrhea, fevers or chills. As mentioned previously, the patient's oncological history is significant for early stage squamous head and neck cancer which was 
in remission since $10 / 2012$. Our patient was initially diagnosed with RCC in October 2013, followed by a right nephroureterectomy in the sameyear. Unfortunately within the nextyear, he was found to have metastatic renal disease and he was started on Sunitinib (vascular growth factor inhibitor) with a follow-up CT scan in June 2014 demonstrating progression in metastatic disease with involvement of lung and retroperitoneum. The patient's treatment plan was palliative and consisted of 2 nd line Nivolumab to be given intravenously every two weeks until disease progression.

The patient was admitted to the hospital with admission showing a potassium of 5.8, phosphorus of 4.6, uric acid of 10.8, and calcium of 8.6 which met the criteria for Cairo-Bishop definition of laboratory TLS. Along with the TLS labs mentioned, patient was also noted to have an acute kidney injury with his BUN/Cr increased to 37/2.51 respectively from a baseline $\mathrm{Cr}$ of $1.1-1.3$ and transaminases with liver enzymes slightly increased from $31 / 18$ to $194 / 159$. He was treated with fluids, insulin, glucose, and allopurinol. He was subsequently transferred to the Intensive Care Unit (ICU) due to labile blood pressure non-responsive to IVF, oliguric renal failure, tumor lysis syndrome and possible sepsis. During the work-up in the ICU, autoimmune processes involving adrenal glands were considered as possible causes of the hypotension. Severe adrenal insufficiency was later ruled out due to lab findings showing AM cortisol levels of 15 and 16 on two occasions, which are relatively low during times of stress. Thyroid function was uncontrolled throughout the ICU state with TSH levels at 9, which had decreased from 12 at admission after adjustment of levothyroxine dose. The patient was placed on stress dose of steroids, Cefepime, and vasopressors with slight improvement in urinary output and hypotension. He was intubated meanwhile for altered mental status and acute respiratory failure and persistent tachycardia. At this point all cultures were negative and there was no evident source of infection. Tumor lysis labs all normalized, with his potassium noted to be 3.3 , phosphorus 3.4 , uric acid 2.0, and calcium 7.7. Patients liver functions tests also were noted to be improve with last documented values showing AST/ALT of 49/119. Patients renal function continued to deteriorate with BUN/Cr on day of death noted to be $67 / 3.48$. Weaning from vasopressor treatment was attempted once during his ICU stay, however due to persistent hypotension, vasopressor requirement increased substantially and continued throughout his admission, until patient's family decided to pursue palliative measures. After one week the patient status deteriorated and he expired while still intubated. Cytokine levels were not further explored due to his death and respecting family wishes.

\section{Discussion}

Tumor Lysis Syndrome is usually rare in solid tumors and is most often associated with metastatic cancer and treatment with chemotherapy. A current literature review describes four cases of TLS in solid renal cancers including two clear cell carcinomas, one transitional cell carcinoma, and one chromophobe cancer, which were all metastatic cases. ${ }^{7}$ TLS occurred following the administration of chemotherapy or targeted therapy in each of these cases. The diagnosis of TLS is straightforward in the aforementioned case presentation. According to the Cairo Bishop definition. ${ }^{8}$ Tumor Lysis Syndrome is when two or more laboratory changes occur within three days before or seven days after cytotoxic therapy. Our patient met the criteria based on laboratory findings after he received the first dose of Nivolumab.

Although our patient clearly had TLS, previous studies have not shown a significant association of TLS with Nivolumab. Tumor lysis syndrome with immune checkpoint inhibitors has never been described so far. The probability of appearance of TLS after the first infusion of Nivolumab seems to be highly unlikely in regard to the mode of action as this drug does not induce direct cell death. Lastly, the clinical course with rapid deterioration and cardiac dysfunction with hypotension makes it very likely that there was another reason for this course. Based on the abnormal levels of thyroid hormones and the hypotension we diagnosed an immune-related toxicity, probably a cytokine storm. Cytokine storms are not reported with immune checkpoint inhibitors but they were reported with CAR-T cells, another form of immunotherapy. Phase II trials program (from Juno Therapeutics) was suspended by the FDA after 3 deaths, which could be treatment-related..$^{9,10}$

Two phase II trials ${ }^{11}$ have yielded positive results with the use of Nivolumab in metastatic RCC and other refractory or recurrent malignancies showing opportunity for expanding uses of this immunotherapy. A phase III trial. ${ }^{6}$ (CheckMate -025) in 821 patients with metastatic RCC showed an overall survival gain of about 6months compared to Everolimus (VEGF inhibitor) . ${ }^{6}$ However, none of these studies reported TLS as one of the side effects observed in each respective sample group. In Checkmate 025, the most common adverse reactions $(\geq 20 \%)$ reported in patients receiving Nivolumab vs Everolimus were fatigue ( $56 \%$ vs $57 \%$ ), cough ( $34 \%$ vs $38 \%$ ), nausea ( $28 \%$ vs $29 \%$ ), rash ( $28 \%$ vs $36 \%$ ), dyspnea ( $27 \%$ vs $31 \%$ ), diarrhea ( $25 \%$ vs $32 \%)$, constipation ( $23 \%$ vs $18 \%)$, decreased appetite $(23 \%$ vs $30 \%)$, back pain ( $21 \%$ vs $16 \%)$, and arthralgia ( $20 \%$ vs $14 \%)$. This case-report is the first observation of an autoimmune or inflammatory syndrome mimicking TLS after one dose of an immunotherapeutic agent as Nivolumab. ${ }^{12}$

\section{Conclusion}

This case report describes an individual with metastatic RCC who received palliative treatment with Nivolumab and subsequently developed an autoimmune/inflammatory process presenting as tumor lysis syndrome leading to his death 9days following initiation of immunotherapy. This report highlights the need for careful follow-up and further research on the possible association between Nivolumab and other immunotherapeutic agents with TLS and auto-immune crisis

\section{Consent for publication}

A formal consent was obtained from the patient's next of kin (spouse) by Dr. Reena M. Patel and documented in electronic medical chart on February 23, 2016 at 11:23 am.

\section{Availability of data and material}

Data sharing not applicable to this article as no datasets were generated or analyzed during the current report.

\section{Conflicts of Interest}

Authors declare that they have no conflict of interest.

\section{Authors' contributions}

HS is the principle investigator for this case report. He wrote the manuscript assisted by BS, RP and JP. HS and JP oversaw all major components of the case report. RP and BS assisted in literature review and drafting part of the case report as well. All authors read and approved the final case report. 


\section{Acknowledgments}

Not applicable.

\section{Conflicts of Interest}

Author declares there are no conflicts of interest.

\section{Funding}

None.

\section{References}

1. Coiffier B, Altman A, Pui CH, et al. Guidelines for the management of pediatric and adult tumor lysis syndrome:an evidence-based review. Journal of Clinical Oncology. 2008;26(16):2767-2778.

2. Mirrakhimov AE, Ali AM, Khan M, Barbaryan A. Tumor lysis syndrome in solid tumors:an up to date review of the literature. Rare Tumors. 2014;6(2):5389.

3. McBride A, Westervelt P. Recognizing and managing the expanded risk of tumor lysis syndrome in hematologic and solid malignancies. $J$ Hematol Oncol. 2012;5(75):75.

4. Znaor A, Lortet-Tieulent J, Laversanne M, et al. International variations and trends in renal cell carcinoma incidence and mortality. European Urology. 2015;67(3):519-530.
5. Albiges L, Choueiri T, Escudier B, et al. A systematic review of sequencing and combinations of systemic therapy in metastatic renal cancer. European Urology. 2015;67(1):100-110.

6. Tomita Y, Fukasawa S, Shinohara N, et al. Nivolumab versus everolimus in advanced renal-cell carcinoma. New England Journal of Medicine. 2015;373(19):1803-1813.

7. Mirrakhimov AE, Voore P, Khan M, et al. Tumor lysis syndrome: A clinical review. World J Crit Care Med. 2015;4(2):130.

8. Cairo MS, Bishop M. Tumour lysis syndrome: new therapeutic strategies and classification. Br J Haematol. 2004;127(1):3-11.

9. Gameiro DN (2006) UPDATED: Storm on the CAR-T field - 3 patients die in Phase II Study.

10. Xu XJ, Tang YM. Cytokine release syndrome in cancer immunotherapy with chimeric antigen receptor engineered $\mathrm{T}$ cells. Cancer lett. 2014;343(2):172-178.

11. Parekh H, Rini BI. Emerging therapeutic approaches in renal cell carcinoma. Expert review of anticancer therapy. 2015;15(11):13051314 .

12. Rizvi NA, Mazières $J$, Planchard $D$, et al. Activity and safety of nivolumab, an anti-PD-1 immune checkpoint inhibitor, for patients with advanced, refractory squamous non-small-cell lung cancer (CheckMate 063): a phase 2, single-arm trial. Lancet Oncol. 2015;16(3):257-265. 